\title{
Consensus decision making in human crowds
}

\author{
JOHN R. G. DYER*, CHRISTOS C. IOANNOU*, LESLEY J. MORRELL*, DARREN P. CROFT*†, \\ IAIN D. COUZIN $\$ \S$, DEAN A. WATERS* \& JENS KRAUSE* \\ *Institute of Integrative and Comparative Biology, University of Leeds \\ $\dagger$ School of Biological Sciences, University of Wales Bangor \\ $\ddagger$ Department of Zoology, University of Oxford \\ $\S$ Department of Ecology and Evolutionary Biology, Princeton University
}

\begin{abstract}
In groups of animals only a small proportion of individuals may possess particular information, such as a migration route or the direction to a resource. Individuals may differ in preferred direction resulting in conflicts of interest and, therefore, consensus decisions may have to be made to prevent the group from splitting. Recent theoretical work has shown how leadership and consensus decision making can occur without active signalling or individual recognition. Here we test these predictions experimentally using humans. We found that a small informed minority could guide a group of naive individuals to a target without verbal communication or obvious signalling. Both the time to target and deviation from target were decreased by the presence of informed individuals. When conflicting directional information was given to different group members, the time taken to reach the target was not significantly increased; suggesting that consensus decision making in conflict situations is possible, and highly efficient. Where there was imbalance in the number of informed individuals with conflicting information, the majority dictated group direction. Our results also suggest that the spatial starting position of informed individuals influences group motion, which has implications in terms of crowd control and planning for evacuations.
\end{abstract}

Animals that form groups frequently have to make important consensus decisions regarding their direction of locomotion, the activities they perform and the timing and duration of these activities (Conradt \& Roper 2003). In some cases, it has been shown that very few individuals within a group may actually possess information on, for example, the direction to a resource (ants, Leptothorax albipennis: Franks et al. 2002; honeybees, Apis mellifera: Seeley 2003; golden shiners, Notemigonus crysoleucas (in the laboratory): Reebs 2000; guppies, Poecilia reticulata (in the laboratory): Reader \& Laland 2000; Swaney et al. 2001). The simplest situation where such information differences

Correspondence and present address: J. Dyer, Institute of Integrative and Comparative Biology, University of Leeds, Leeds LS2 9JT, U.K. (email: bgyjdd@leeds.ac.uk).D. P. Croft is at the School of Biological Sciences, University of Wales Bangor, Deiniol Road, Bangor, Gwynedd LL57 2UW, U.K. I. D. Couzin is at the Department of Ecology and Evolutionary Biology, Princeton University, Princeton, NJ 08544-1003, U.S.A. among group members are present could be because of the different spatial positions of individuals. For example, peripheral group members are likely to encounter a stimulus first (a predator for example) and will respond accordingly, the information, such as a change in direction, may then spread through the rest of the group (Radakov 1973; Kils 1986). Information differences may also occur as a result of differences in experience and learning between individuals. In some migrating flocks of birds, it has been shown that younger inexperienced individuals follow older more experienced birds that have previously travelled the route (Lovvorn \& Kirkpatrick 1982; Maransky \& Bildstein 2001). Some individuals may have encountered and learnt to recognize certain predators and respond with evasive behaviour, which is likely to spread through the group including to those which have no previous experience of the predator (Radakov 1973).

In many situations conflicts may exist between the preferences of different group members (Conradt 1998; Ruckstuhl 1998; Ruckstuhl \& Neuhaus 2000; 
Ruckstuhl \& Neuhaus 2002; Couzin et al. 2005). If these conflicts cannot be resolved and no consensus can be reached then the group will split and individuals will forego the benefits associated with being part of a large group (Krause \& Ruxton 2002). Consequently, animals that benefit greatly from being part of a large group are likely to have evolved mechanisms by which conflicts in individual preferences can be quickly and efficiently resolved, thereby maintaining group cohesion.

Modelling work has provided some insight into the functions and mechanisms of collective behaviour and consensus decision making in animal groups. Conradt \& Roper (2003) investigated the relative fitness consequences of democratic and despotic decisions, finding that in most situations democratic decisions result in less extreme outcomes that are likely to be less costly to the group as a whole. They conclude that democratic decisions should be widespread in the animal kingdom and that despotic decisions should only be beneficial where groups are small and differences in information are large.

Couzin et al. (2005) used computer simulations to investigate the mechanisms behind collective behaviour and consensus decision making. They showed that only a very small proportion of informed individuals are required to guide a group primarily composed of naive individuals towards a target location. They found that consensus decisions can be reached even when the informed individuals do not know whether they are in a majority or minority, or how the information they possess compares with that of other informed individuals with potentially conflicting information. While this model provides unique insight into decision-making processes in animal groups, as yet these predictions remain largely untested.

Empirical work on consensus decision making has primarily concentrated on the eusocial insects (e.g. Camazine et al. 1999; Seeley \& Buhrman 1999; Mallon et al. 2001; Britton et al. 2002; Pratt et al. 2002; Couzin $\&$ Franks 2003; Franks et al. 2003; Seeley 2003; Dornhaus et al. 2004; Seeley \& Visscher 2004; Pratt 2005) and, therefore, much more is known about the mechanisms behind consensus decisions in eusocial insect colonies than in vertebrate groups. Two particularly well studied and elegant examples involve nest site choice in swarming honeybees, $A$. mellifera, and the ant, L. albipennis. Scouts locate potential new sites and their recruiting success is dependent on the quality of the new site (honeybees, A. mellifera: Seeley \& Buhrman 1999; Seeley 2003; Seeley \& Visscher 2004; ants, L. albipennis: Mallon et al. 2001; Franks et al. 2003). This positive feedback loop ensures that consensus decision can be achieved when the number of scouts recruiting for a particular site reaches a certain threshold (quorum), at which point all individuals leave for the new site (Britton et al. 2002; Franks et al. 2003). In both examples the underlying mechanism is based on simple rules of self-organization (Camazine et al. 2001; Conradt \& Roper 2005).

Work on consensus decision making in vertebrate groups has largely concentrated on decisions about travel routes or the timing of activities (Conradt \& Roper 2005).
Traditionally, it was thought that in vertebrate groups, dominant individuals would lead decision making and largely determine the outcome. More recently, research has suggested that consensus decisions may be more common than previously thought (Conradt \& Roper 2003). Examples of consensus decisions in vertebrate groups often involve the group commencing movement or moving in a particular direction when a certain threshold of individuals have signalled their intent through means such as head movements (Whooper swans, Cygnus cygnus: Black 1988), calling (Gorillas, Gorilla gorilla: Stewart \& Harcourt 1994) or gazing in a particular direction (African Buffalo, Syncerus caffer: Prins 1996). Reebs (2000) showed that a small minority of informed fish (golden shiners, Notemigonus crysoleucas) can guide naïve conspecifics from a preferred area of the tank, to a less preferred brightly lit area of the tank where food was expected. Reader et al. (2003) found that four demonstrator guppies, P. reticulata, could guide four naive conspecifics through a hole to escape an oncoming trawl net. In this example, all individuals shared the motivation to escape the trawl net, but only some of them knew the correct escape route.

Small groups are widely used to make important decisions in human societies (Davis 1992a; Winquist \& Larson 1998; Kocher \& Sutter 2005). Consequently, decision making in human groups has been, and continues to be, an important topic for both social psychologists (see McGrath 1978; Brandstatter et al. 1982, Levine \& Moreland 1990; Davis 1992b; Ten Velden et al. 2007) and economists (e.g. Bornstein \& Yaniv 1998; Kocher \& Sutter 2005; Eliaza et al. 2007). A large body of work has looked at both the outcomes of group decision making, often through comparing group decisions with individual decisions (e.g. Bornstein \& Yaniv 1998; Kocher \& Sutter 2005), and the processes involved in group decision making, including the influence of majorities and minorities on group decisions (e.g. Asch 1956; Latané \& Wolf 1981; Ten Velden et al. 2007). Asch (1956) famously showed that individuals would conform to a group even when they knew they were wrong, demonstrating the power of majority influence in groups. A wealth of psychology literature also exists on leadership and followership behaviour, which is reviewed from an evolutionary perspective by Van Vugt (2006).

Most research into group motion in human crowds has been largely theoretical and has mainly concentrated on modelling pedestrian behaviour in evacuation scenarios. Influential research by Helbing et al. (2000) specifically investigated the mechanisms and conditions that lead to panic and jamming through uncoordinated motion in human crowds. They found that a mixture of individualistic and collective behaviour is the optimal strategy for escaping a smoke filled room. Individualistic behaviour allowed some individuals to successfully locate exits, and collective (herding) behaviour ensured that the behaviour was imitated.

Aube \& Shield (2004) also modelled an evacuation scenario to investigate the effect of placing informed leader agents at different points in a panicking crowd, finding that a mixture of embedded, peripheral and distant leaders is the most effective way of saving the greatest number of individuals in the shortest time. 
This study uses human groups to investigate consensus decision making both in the absence and presence of conflicting information. First, we look at how the proportion of informed individuals within a group affects the speed and accuracy with which a consensus decision is reached. Couzin et al. (2005) predict that for a given group size accuracy increases asymptotically as the proportion of informed individuals increases. Couzin et al.'s (2005) model does not make predictions about time as no end targets are specified just preferred directions, but we predict that the time taken to reach a target will decrease with an increasing proportion of informed individuals. Second, we introduce conflicting information to different individuals and look at how the conflict is resolved and whether the group can still reach a consensus decision accurately and efficiently. We also examine some of the mechanisms behind the decision-making process. To our knowledge, this is the first time any study on vertebrates has looked at whether groups can overcome conflicts in directional preferences to reach a consensus movement decision.

\section{METHODS}

\section{General Methods}

The experiments took place between February and July 2005 at the University of Leeds and the University of Hull. Participants were undergraduate students, open-day visitors and local school children (aged $14-16$ years). In total, 40 mixed-sex groups of eight individuals were used for testing (20 in the single-target experiment and 20 in the conflict experiment). All participants were naïve as to the purpose of the experiment.

A circular arena with a $10 \mathrm{~m}$ diameter was marked on the floor and cards labelled 1-16 were spaced equally around its perimeter. A circle with a diameter of $2 \mathrm{~m}$ was marked out in the centre of the first circle with the letters $\mathrm{A}-\mathrm{H}$ spaced equally around its perimeter (Fig. 1, inset). Individuals were asked to stand on a letter $(\mathrm{A}-\mathrm{H})$ on the inner circle to ensure that all starting positions were equal and equidistant from the outer periphery. To avoid any bias due to initial direction of locomotion, the initial orientation of each individual in a trial was randomized by instructing them to face a number from the outer circle chosen at random without replacement.

Each group was given the following standard set of instructions: 'When we tell you to begin you should start walking at a normal speed and do not stop before being told to do so. You can walk anywhere inside or outside the circle but you must remain together as a group of eight and you should not talk or gesture to each other.' Normal walking speed was not defined but was demonstrated to participants. Group splits were observed on three occasions within the conflict treatments. When this occurred it was recorded and the group was reset and reminded that they must stay together, before repeating the trial.

In addition to these standard instructions participants were each handed a slip of paper with an individual behavioural rule to follow. They were instructed to read and memorize the information, then hide the slip to ensure that no other member of the group could see it. The slips of paper gave one of two different behavioural rules, one for uninformed individuals (naïve individuals) and one for informed individuals. Behavioural rule 1 gave instructions to simply 'stay with the group,' resulting in naive individuals. Behavioural rule 2 gave instructions to 'go to number $X$, without leaving the group' creating informed individuals ( $\mathrm{X}$ represents a randomly chosen number on the outer circle between 1 and 16).

\section{Single-Target Experiment}

Each group of eight was tested in three different treatments in which only the number of informed individuals was changed. The treatments were 'control' (all individuals receive behavioural rule 1), 'one-informed' (one individual receives behavioural rule 2 , the rest receive behavioural rule 1), and 'two-informed' (two individuals receive behavioural rule 2 , the rest receive behavioural rule 1). For each group of eight, two-informed individuals were randomly selected from the eight starting positions. The first was used in the one-informed treatment and both were used in the two-informed treatment to reduce variability between different treatments of the same group.

The informed individuals' targets were randomly assigned and in the two-informed treatment both the informed individuals were given the same target number on their instruction slip. Because of our repeated-measures design there was a potential for order (training) effects within groups. To minimize these effects the six possible orders of the three treatments were systematically tested. Our subjects were not allowed to talk or gesture during the experiments. This condition may not be unrealistic for humans in crowds given that our trials are usually very short (median time of $15.5 \mathrm{~s}$ ) and that strangers are usually at least initially reluctant to actively exchange information.

Each trial lasted until any member of the group came within approximately $50 \mathrm{~cm}$ of the outer circle as judged by one of two observers, placed on either side of the circle and if no target was reached within $3 \mathrm{~min}$, the trial was terminated. The observers had no information on which treatment was being tested, who was informed, or what their target was. For each trial, we recorded the time taken to get to within approximately $50 \mathrm{~cm}$ of the perimeter and the number closest to the groups' finishing position.

\section{Conflict Experiment}

For the second experiment, a conflict in the information given to the informed individuals was introduced. The experimental arena for the conflict experiments was identical to the single-target experiment. The informed individuals were given one of two separate targets, $180^{\circ}$ apart. We used four treatments, which differed in the number of individuals given each target: in the first treatment, two individuals were given each target (the ' 2 versus 2 ' treatment), in the second, three individuals were given one target, and two the second (3 versus 2), in the third, four individuals were given one target and two the second (4 versus 2 ), and in the fourth, three individuals were given one target and one the second 


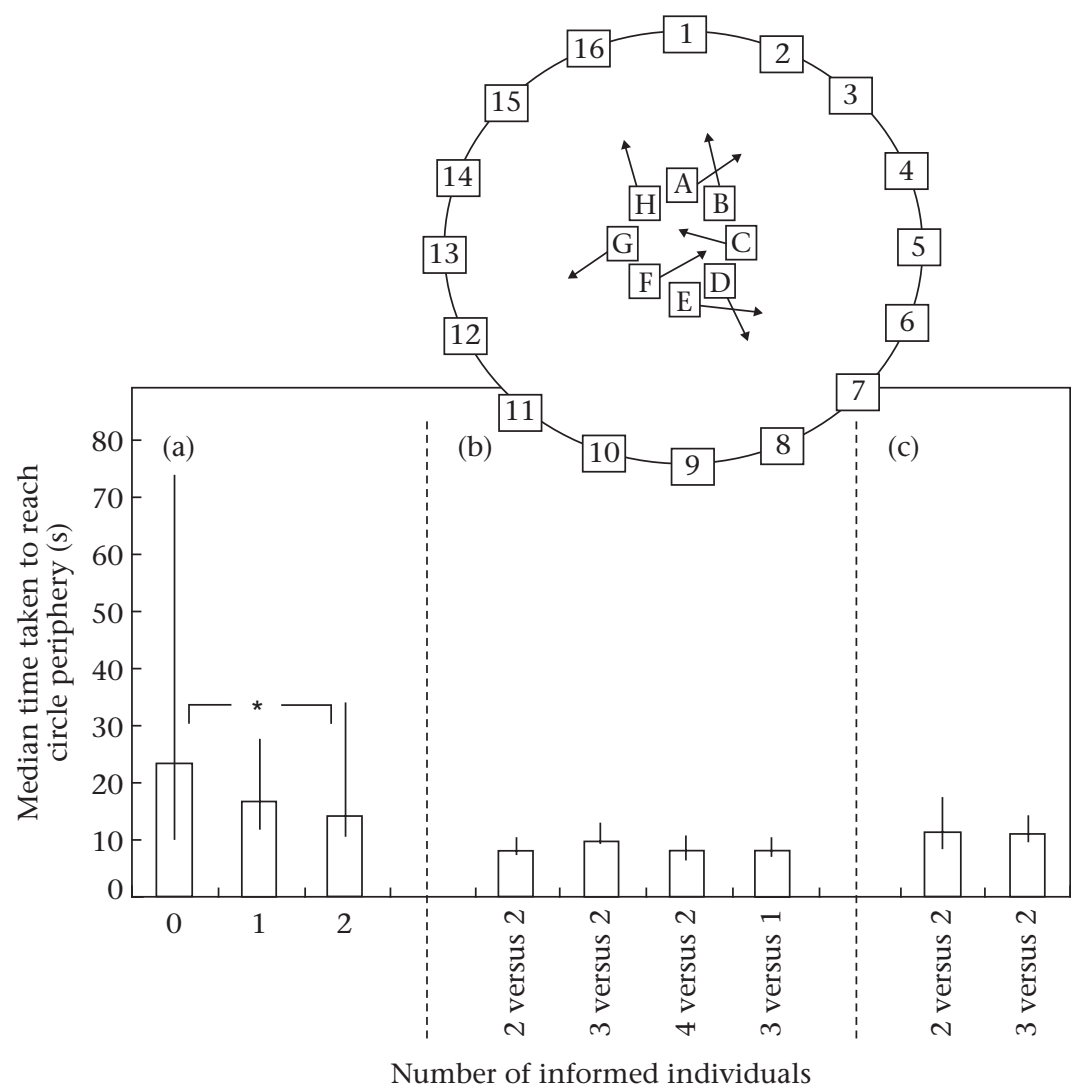

Figure 1. Median ( \pm quartiles) time taken to reach the periphery of the circle by groups tested under the different experimental treatments. (a) The single-target treatments: zero, one and two informed. Treatment differences are indicated by Friedman post hoc tests. Asterisk denotes a significance of 0.025 (see Conover 1999). (b) The conflict treatments: 2 versus 2, 3 versus 2, 4 versus 2, and 3 versus 1 ( $N=12$ ). (c) The conflict treatments: 2 versus 2 and 3 versus 2 with their greater sample size $(N=20)$. (Inset) Overhead view of the arena (diameter $10 \mathrm{~m}$ ). The letters forming the inner circle denote the start positions of individuals and the arrows their respective start orientation. The numbers on the outer circle were used (1) to orientate the individuals at the start of each trial and (2) as target destinations for leaders.

(3 versus 1). This allowed us to test the prediction of Couzin et al. (2005) that even a small inequality in the numbers of individuals in conflict will result in the majority deciding the direction of group motion.

As before, we used a repeated-measures design, where each group of eight individuals was tested in each of the four treatments. Informed individuals were randomly assigned for the 2 versus 2 treatment and similarly to the single-target experiment these same individuals were the informed individuals in each of the other treatments but with one extra and two extra informed individuals randomly assigned to the 3 versus 2 and 4 versus 2 treatments, respectively. This allowed us to minimize the potential for individual behavioural variation to affect the results for a given group. As before, the different treatment orders were tested systematically.

At the start of our investigation, we used only the 2 versus 2 and 3 versus 2 treatments, thus the first eight groups were only tested in these two treatments. Subsequently, we added the 4 versus 2 treatment (to increase the imbalance in the conflict) and the 3 versus 1 treatment (as a contrast to 2 versus 2 controlling for absolute number of informed individuals) and the 12 remaining groups were tested in all four treatments. Participants in the conflict experiment had not taken part in the single-target experiment.
Trials followed the same format as for the single-target experiment. From the information on the final finishing position of each group, we calculated a measure of deviation, based on how the final position differed from the closest of the two target locations.

Where the number of individuals with each target was equal (2 versus 2 treatment) we looked at whether the spatial starting positions of informed individuals could influence the overall direction of the group. First, for each pair of informed individuals with a common target we calculated the number of individuals separating them from each other. Second, we calculated the difference between the separation values of each pair and looked at how this affected the overall direction of the group. We hypothesize that as the difference between the separation values increases the more likely it is that the pair that starts closer together will determine the overall group direction. We judged that one of the pairs has determined group direction if the group finishes at the target of that pair, or within two numbers either side.

\section{The Effect of Previously Informed Individuals}

In some situations (e.g. if the two-informed trial came before the one-informed trial, or in the conflict experiment, 
if the 4 versus 2 trial came before the 2 versus 2 trial) individuals who were informed in the previous trial would be naive in the following trial. We test for the effects of previously informed naive individuals for each treatment separately within the single-target and conflict experiments. This is done by comparing the time to periphery and deviation from target on occasions where previously informed (now naïve) individuals were present and occasions were they were not. For example for some groups, the one-informed trial preceded the twoinformed trial and for others the opposite was true. Comparison between these situations allows us to determine whether previously informed individuals had a significant effect. This analysis is possible for the control treatment (compare cases where one or two individuals were previously informed to cases where none were previously informed), the one-informed treatment (compare cases where one individual was previously informed to cases where none were), the 2 versus 2 and 3 versus 1 treatments (as for control treatment), and the 3 versus 2 treatment (as for one-informed treatment).

\section{Video Analysis}

To investigate the mechanisms of leadership, where possible (9 of the 20 single-target groups) groups were recorded on video film from a stage raised above the arena. The video was examined to discover how the behaviour of informed individuals differed from that of the naïve group members. First, we looked at whether the informed individuals spent more time in the vicinity of their target than uniformed individuals. We divided the arena into quarters, with the target always at the centre of one of the quarters (the target quarter). For each group, we recorded how much time each individual spent in the target quarter. The trials from the one-informed treatment were analysed and on occasions where the one-informed groups could not be used (i.e. they did not reach or get near to their target) we used the two-informed trial from the same group. To analyse the results we calculated the mean time spent in the target quarter by the uninformed individuals and compared this with the time spent in the target quarter by the informed individual, or the mean of the time spent in the target quartile by the informed individuals, where two-informed trials were used.

Secondly, we looked at whether informed individuals spent more time at the front of the group. We measured this from the point where the group polarized, which was judged by two individuals independently as the point where all individuals within the group were facing and walking in a uniform direction towards the target. From this point to the end of the trial we measured the length of time spent at the front of the group by the informed individual compared with the mean time spent at the front by uninformed group members.

\section{Statistical Analyses}

Statistical analysis was carried out in SPSS (SPSS Inc., Chicago, IL, U.S.A.), version 11. A binary logistic regression was used to analyse the data on the effect of spatial starting position of leaders on the likelihood of them determining group direction. Otherwise, nonparametric tests were used throughout as none of the data conformed to parametric assumptions, neither could it be satisfactorily transformed. Exact tests were used throughout; therefore, all $P$ values displayed are Pexact. Friedman tests were used to make comparisons for paired data. Where a Friedman test returned a significant $P$ value, we used the post hoc method outlined in Conover (1999) to make multiple comparisons between individual treatments. Mann-Whitney $U$ tests were used for independent data. Spearman correlations and binomial tests were also used.

\section{RESULTS}

\section{Testing for Training Effects}

Because of the repeated-measures design there was a potential for each group's performance to improve with each trial (training effect). We found no significant difference in either the time to periphery and deviation from target between groups in their first, second and third trials for the single-target experiment (Friedman tests, time: $\chi^{2}=3.44, N=20, P=0.184$; deviation from target: $\chi^{2}=2.39, N=19, P=0.323$ ) and first, second, third and fourth trials for the conflict experiment (Friedman tests, time: $\chi^{2}=5.10, N=12, P=0.167$; deviation from target: $\left.\chi^{2}=3.69, N=12, P=0.299\right)$.

\section{The Effect of Previously Informed Individuals}

There was no significant difference in time taken to reach the periphery, for trials where no previously informed individuals were present and those where one or more previously informed individuals were present, in any of the single-target treatments (Mann-Whitney $U$ tests, control: $Z=-1.043, N=8.12, P=0.314$; one-informed: $Z=-1.635, N=10.9, P=0.108)$ or conflict treatments (2 versus $2: Z=-1.313, N=13.7, P=0.202,3$ versus 2 : $Z=-0.000, N=13.7, P=1.000,3$ versus $1: Z=-1.376$, $N=6.6, P=0.180)$. Likewise, for the one-informed treatment there was no significant difference in deviation from target between trials where no previously informed individuals were present and those where one previously informed individual was present (Mann-Whitney $U$ test, $Z=-1.148, N=10,9, P=0.272$ ). This was not tested for conflict treatments as there were too few occasions when groups deviated from their targets.

\section{Single-Target Experiment}

\section{Time to periphery}

The time taken to reach the periphery differed significantly between treatments (Friedman test: $\chi^{2}=6.08$, $N=20, P=0.046)$. Groups with two-informed individuals were faster to reach the periphery than control groups, but no significant difference was found between either groups 
with one-informed individual and controls, or groups with one and two-informed individuals (Fig. 1a).

\section{Deviation from target}

The deviation from the target location differed significantly between treatments (Friedman test: $\chi^{2}=16.35$, $N=19, P<0.001)$. One of the groups did not reach the periphery of the circle within the maximum $3 \mathrm{~min}$ in the one-informed treatment resulting in a sample size of 19. Both the treatments with one-informed individual and two-informed individuals showed smaller deviations from the target location than the control treatment. However, two-informed individuals did not bring a significant decrease in the deviation from the target location, compared with one-informed individual. This is probably because the accuracy is already very high with just oneinformed individual (Fig. 2a).

\section{Speed versus accuracy}

Finally, we tested whether higher speed (to reach the periphery) came at the expense of lower accuracy. No relationship between the time taken to reach the periphery and deviation from the target location was found within any of the three treatments (Spearman correlation: control: $r_{s}=-0.052, N=20, P=0.828 ; 1$ informed: $r_{s}=-0.76, \quad N=19, P=0.757 ; 2$ informed: $r_{s}=0.217$, $N=20, P=0.358)$.

\section{Conflict Experiment}

\section{Time to periphery}

There was no significant difference in the time taken to reach the periphery of the circle between treatments 2 versus 2,3 versus 2 and 4 versus 2 (Friedman test:

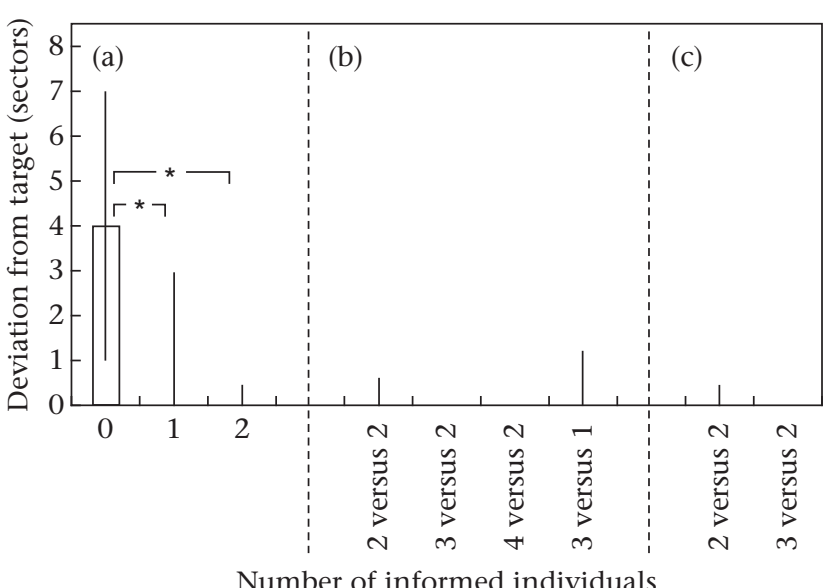

Figure 2. Median ( \pm quartiles) deviation of groups from their target under the different experimental treatments. (a) The single-target treatments: zero, one and two informed. Treatment differences are indicated by Friedman post hoc tests. Asterisk denotes a significance of 0.025 (see Conover 1999). (b) The conflict treatments: 2 versus 2 , 3 versus 2, 4 versus 2, and 3 versus $1(N=12)$. (c) The conflict treatments: 2 versus 2 and 3 versus 2 with their greater sample size $(N=20)$. $\chi^{2}=3.41, N=12, P=0.193$; Fig. $\left.1 b\right)$. There was also no significant difference in time taken between the 2 versus 2 and 3 versus 2 treatments when tested separately using their greater sample size of 20 (Wilcoxon test: $z=-1.02$, $N=20, P=0.322$; Fig. 1c).

\section{Deviation from target}

There was no significant difference between the treatments in the deviation of groups from the target location (Friedman test: $\chi^{2}=4.80, N=12, P=0.123$; Fig. 2b). Median deviation was zero for all four treatments, with groups finishing exactly at their targets in the vast majority of trials (8/12 times in the 2 versus 2 treatment, $10 / 12$ times in the 3 versus 2 treatment, $12 / 12$ times in the 4 versus 2 treatment, and 7/12 times in the 3 versus 1 treatment). There was also no significant difference in deviation between the 2 versus 2 and 3 versus 2 treatments when tested separately using their greater sample size of 20 (Wilcoxon test: $z=-1.38, N=20, P=0.219$; Fig. 2c).

\section{Overall group direction}

One set of informed individuals was determined to have decided overall group direction when the group finished at their target or within two numbers either side of it. In the 3 versus 1 treatment, two of the groups failed to finish within two numbers each side of either target and so neither set of informed individuals was deemed to have determined group direction and $N$ is reduced to 10 . In those treatments where the two groups of informed individuals differed in numbers, the majority won a significant proportion of the decisions regarding the overall group direction (binomial tests: 3 versus $2: N=20, P=0.041$ (75\% of groups finished at majority target, $25 \%$ at minority target); 4 versus $2: N=12, P=0.039$ (83\% of groups finished at majority target, $17 \%$ at minority target); 3 versus 1: $N=10, P=0.021$ (75\% of groups finished at majority target, $8 \%$ at minority target, $17 \%$ at neither target; Fig. 3). The result for the 3 versus 1 treatment shows that the majority leads the group when the total number of informed individuals is controlled for between treatments (in this case a total of four informed individuals for both the 2 versus 2 treatment and 3 versus 1 treatment).

For the treatment of 3 versus 2 deviation from the target was almost always zero regardless of whether overall group direction was dominated by the minority (two-informed individuals, median deviation $=0$, five out of five groups finished exactly at their targets) or the majority (threeinformed individuals, median deviation $=0,13$ out of 15 groups finished exactly at their targets). This result suggests that once a group of informed individuals is in control, they can effectively steer the overall group regardless of whether this is the smaller (e.g. two-informed individuals) or larger group of informed individuals (i.e. threeinformed individuals). Similarly, the groups' progress towards the target was not slower when the minority led the group (two-informed individuals, median time $=9 \mathrm{~s}$ ), than when the majority (three-informed individuals, median time $=11 \mathrm{~s}$ ) was in control of the group (MannWhitney $U$ test: $Z=-0.439, N=15.5, P=0.686)$. We did not have a large enough sample size to test this for the 4 


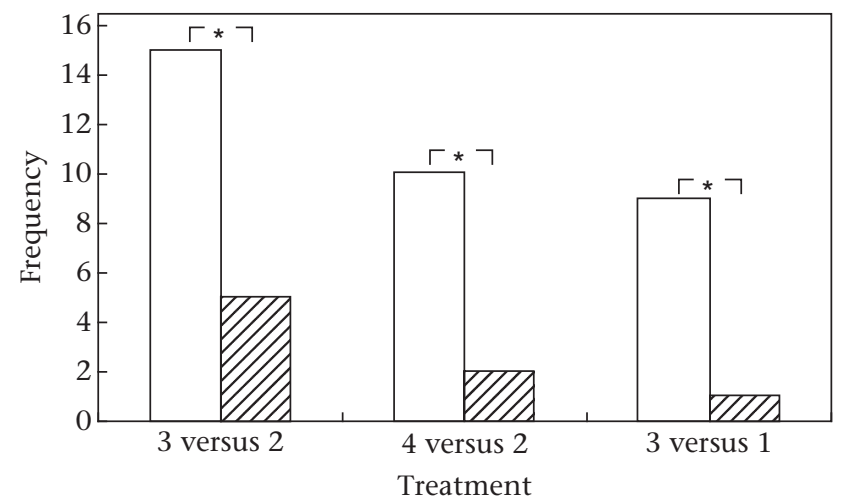

Figure 3. Number of times the majority and minority subsets of informed individuals determined group direction (finishing at their target or within two numbers either side) in the 3 versus 2, 4 versus 2 and 3 versus 1 conflict treatments. There were two occasions where deviation exceeded 2 in the 3 versus 1 treatment and, therefore, $N=10$ for this treatment. Hollow bars represent the majority for each treatment and striped bars represent the minority. Treatment differences are indicated by binomial tests: asterisk denotes a significance of 0.05 .

versus 2 or 3 versus 1 treatments, as there were only two occasions on which the minority dominated the overall direction of the group for the 4 versus 2 treatment and one occasion for the 3 versus 1 treatment.

\section{Speed versus accuracy}

There was clearly no relationship between the time taken to reach the periphery and deviation from the target location within any of the conflict treatments. This was not tested statistically as there were so few occasions when groups deviated at all from their targets.

\section{Spatial starting position of informed individuals}

Where the number of individuals with each target was equal ( 2 versus 2 treatment) we found an influence of the spatial starting positions of informed individuals. As the difference between the separation values (closeness) of the two pairs increased the more likely it was that the pair that started closer together decided the overall group direction (binary logistic regression: $\chi^{2}=7.476$, $N=20, P=0.006)$

\section{Conflict Compared with Single Target}

There was no difference in the time taken to reach the periphery of the circle or the accuracy with which the target was reached between the treatment with two-informed individuals and a single target ( 2 versus 0$)$ and the treatment where two pairs of informed individuals each pulled in different directions ( 2 versus 2; Mann-Whitney $U$ tests, time: $Z=-1.578, N_{1}=N_{2}=20, P=0.114$; Fig. 1 ; deviation from target: $Z=-0.362, \quad N_{1}=N_{2}=20$, $P=0.717$; Fig. 2). This result suggests that collective decisions in conflict situations situation can be made very efficiently.

\section{Individual Level Behaviour}

Informed individuals spent significantly more time in the quartile containing their target than uninformed individuals (informed median (s) $\pm \mathrm{IQR}=10.5 \pm 24$; uninformed median (s) $\pm \mathrm{IQR}=7 \pm 18$.3: Wilcoxon test: $Z=-2.07, N=9, P=0.039$ ). Informed individuals also spent significantly more time at the front of the group than uninformed individuals after the group had polarized (Wilcoxon test: $z=-2.31, N=9, P=0.020$; Fig. 4).

\section{DISCUSSION}

Our results show that, in line with theoretical predictions (Couzin et al. 2005) and empirical studies (Köhler 1976; Brown 1986; Reebs 2000; Brown et al. 2006), a minority of informed individuals can lead a naive group. We found that both the speed and accuracy of human crowd movement were influenced by the presence of informed individuals in the group. The influence of informed individuals on the group direction appeared to be more substantial than the influence on group speed. Across taxa, previous studies have reported a link between information status and leadership. In colonial birds, individuals follow conspecifics that foraged successfully in the recent past (Brown 1986) and in shoaling fish it has been shown that trained individuals could manipulate the direction of the group (Köhler 1976; Reebs 2000; Brown et al. 2006).

We found that neither the speed nor accuracy of the group was significantly increased by the presence of twoinformed individuals when compared with a single informed individual (Fig. 1). In contrast, Reebs (2000) showed that increasing the number of trained individuals in a shoal of fish (golden shiners, Notemigonus crysoleucas) had a strong effect on shoal movement direction. However, he increased the number of leaders from a single trained individual, to three and then five out of 12 , which represents a greater proportion of the group than in our study. Couzin et al. (2005) found that accuracy increased asymptotically with proportion of informed individuals and their predictions suggest that we should observe a difference in accuracy between one- and two-informed individuals in a group of eight. In our study, the group

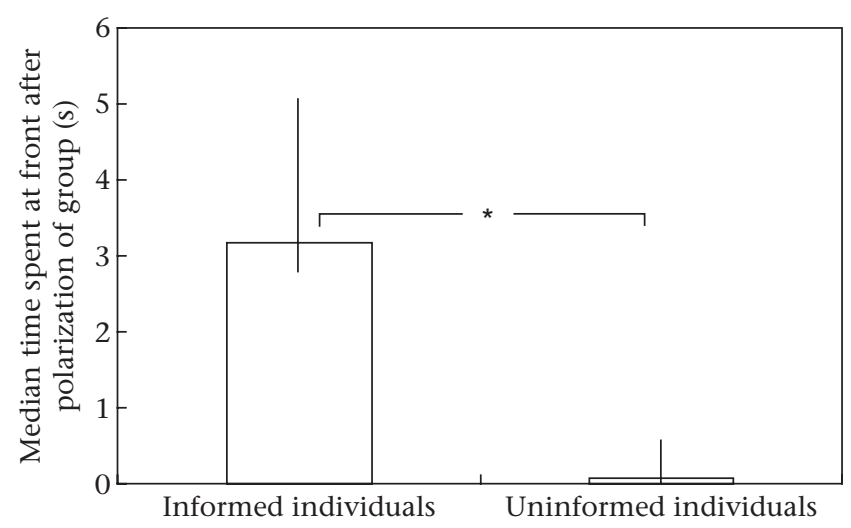

Figure 4. Median ( \pm quartiles) time (s) spent at front by informed individual after polarization of the group. Treatment differences are indicated by Wilcoxon tests: asterisk denotes a significance of 0.05 . 
already achieves very high accuracy with a single informed individual suggesting a more immediate effect. It would be interesting to test the predictions of Couzin et al. (2005) using larger groups and a larger arena to allow for greater variation in the proportion of informed individuals.

It may be predicted that there is a trade-off between the speed and accuracy with which consensus decisions are made: the longer it takes to reach the consensus decision the more likely it is that the decision is accurate. Franks et al. (2003) found that in house-hunting ant colonies, Leptothorax albipennis, a speed-accuracy trade-off exists, and that when conditions are benign the ants take longer over their collective decision making to maximize accuracy, whereas when conditions are harsh they must come to a decision more quickly at the expense of accuracy. We did not find a relationship between the speed and accuracy of our groups. It is possible that our arena was not large enough to allow for sufficient variation between trials or that our sample size was too small to detect such a trade-off.

To our knowledge, the influence of conflicting information on consensus decision making has not previously been tested experimentally. In conflict situations where the number of informed individuals on either side of the conflict differed, the group almost always decided in favour of the majority (e.g. the three in the 3 versus 2 and 3 versus 1 treatments and the four in the 4 versus 2 treatment). This shows that a difference of just one-informed individual can make a decisive difference in terms of the outcome of the consensus decision, as predicted by Couzin et al. (2005). It is probable that in most situations the greater the number of individuals possessing a piece of information, the more likely that information is reliable.

The introduction of conflict into our groups did not significantly increase the time taken to reach the target suggesting that consensus decision making in conflict situations can be remarkably efficient. It is likely that the conflict is resolved very early on. For example, one set of informed individuals may have a more favourable spatial starting position and once they establish control of group motion their preference may be reinforced and the group gains too much momentum for individuals with other preferences to do anything but follow. In one of the variants of the model presented by Couzin et al. (2005) a feedback loop was introduced on the weighting of the preferred direction of informed individuals. If individuals found themselves moving in a similar direction to their preferred direction, their preference for that direction was further reinforced. Likewise if informed individuals are heading away from their preferred direction their preference is reduced.

Modelling work by Aube \& Shield (2004) suggested that the spatial placement of leaders within a crowd could strongly influence the speed and accuracy of group movement. We found that the informed individuals that started the experiment closer together (in trials where the number of informed individuals going to each target was equal) were more likely to determine the direction of the group. Similarly, Leca et al. (2003) found that in groups of white-faced capuchin monkeys, Cebus capucinus, individuals starting in core positions within the group were more likely to successfully initiate group movements than those starting from peripheral positions suggesting that spatial position can be important.

We believe the scenarios we tested can be likened to those where human crowds try to exit buildings during an evacuation because of fire or another hazard, similar to the scenarios modelled by Helbing et al. (2000) and Aube \& Shield (2004). These are situations in which often only a few individuals are informed about the safe location for assembly and there can be considerable background noise which makes verbal information exchange difficult. There are obvious differences in that in our study there was no obvious motivation for naïve individuals to move anywhere or the great degree of panic that could be present. Despite this we hypothesize that very similar basic processes would be likely to govern the movement of the group. Our study and further empirical work looking at group movement in humans could have very important implications in terms of crowd control and in planning for evacuations. This could be in terms of deciding how many individuals need to be informed on locations of exits in confined public spaces and where in the crowd would be the most effective place for informed individuals to be to effectively lead the group. Our finding that the group decides in favour of the majority could potentially mean that in situations where several exits are available and known by various numbers of people, there might result some crowding at one exit while other exits remain unused, although this is true only if there is an incentive for the crowd not to split.

Our analysis of individual level behaviour shows that informed individuals spent longer in the zone containing their target and more time at the front of the group once the group has polarized. This suggests that when the group is heading away from the target, the informed individual stays towards the rear of the group, potentially holding the group back and that when the group moves back towards the target the informed individual attempts to lead the group by moving to the front and taking advantage of the momentum towards the target. Interestingly, our behavioural rules (either 'stay with the group' or 'go to number $X$, without leaving the group') did not specifically code for any group-level pattern or behaviour, yet we find that groups with an informed individual tend to polarize and approach the target with the informed individual at the front of the group. This contrasts to the behaviour of the groups without informed individuals, where a lot of time appeared to be spent circling in a torus (as found in Couzin et al. 2005).

In this study we tried to minimize communication between individuals through obvious signals like talking and pointing. Despite these limitations upon communication between individuals, it was clear that informed individuals often behaved in a way which may have given subtle cues to uninformed individuals. By far the most commonly observed of these behaviours was for informed individuals at the front or on the edge of the group to regularly glance over their shoulders at the group. Other behaviours that we observed were for informed individuals to walk backwards towards the target while facing the group, walking round the edge or through the centre of the 
group to get to the front (in relation to the target) and walking back and forth along the edge of the group regularly changing direction while staying as close as possible to the target. Occasionally, informed individuals were also observed to walk slightly faster than other group members to reach the front of the group. Informed individuals varied greatly in their ability to gain control of group motion, which may have been because of a variety of factors including personality (some individuals may simply have been more confident leading the group) or how they balanced the two different parts of their behavioural rule (go to number $X$, without leaving the group). Although not explicitly tested (because of sample size), we did not observe any obvious gender differences in leadership ability in our study. It may be possible that one sex is more successful at leading or that naive individuals are more likely to follow members of the same or even the opposite sex. Further work could look at the effect of leader gender and personality on group movement decisions and also the influence of changing the relative importance of the different rules. Couzin et al. (2005) found that in their model the balance between the motivation of informed individuals to move in a certain direction and their desire to maintain social cohesion could affect both the accuracy of the group and the likelihood of it splitting.

Our study provides new insight into the ability of informed individuals to lead groups of naive individuals and the ability of groups to resolve conflicts in information. We show for the first time for vertebrate groups, that conflicts in information can be resolved quickly and efficiently, with groups nearly always deciding in favour of the majority. This occurs without the use of obvious signals and without individuals having any knowledge of the quality of any other group members' information or how it compares with their own. Understanding the mechanisms by which knowledgeable individuals can lead naïve groups, and by which consensus decisions can be reached when individuals have conflicting preferences is important in increasing our understanding of group cohesion and may provide insight into the evolution of cooperation.

\section{Acknowledgments}

We would like to thank Dick James, Stefan Krause, Chantima Piyapong, Yan Wong, Marc Botham, Philip Thomas, Ben Chapman, Andrew Whitworth, Anita Glover, Pedro Pedro, Jane Staveley, William Butterworth, Nick Bland, Beth Mcclymont, Karen Cooper and to all volunteers who participated in the experiments. Funding was provided by the Engineering and Physical Sciences Research Council.

\section{References}

Asch, S. E. 1956. Studies of independence and conformity: a minority of one against a unanimous majority. Psychological Monographs, 70, 416

Aube, F. \& Shield, R. 2004. Modelling the effect of leadership on crowd flow dynamics. Cellular Automata, Proceedings, 3305, 601-611.
Black, J. M. 1988. Preflight signalling in swans - a mechanism for group cohesion and flock formation. Ethology, 79, 143-157.

Bornstein, G. \& Yaniv, I. 1998. Individual and group behavior in the ultimatum game: are groups more "rational" players? Experimental Economics, 1, 101-108.

Brandstatter, H., Davis, J. H. \& Stocker-Kreichgauer, G. 1982. Group Decision Making. London: Academic Press.

Britton, N. F., Franks, N. R., Pratt, S. C. \& Seeley, T. D. 2002. Deciding on a new home: how do honeybees agree? Proceedings of the Royal Society of London, Series B, 269, 1383-1388.

Brown, C. R. 1986. Cliff swallow colonies as information centers. Science, 234, 83-85.

Brown, C., Laland, K. \& Krause, J. 2006. Fish Cognition and Behaviour. Oxford: Blackwell Scientific.

Camazine, S., Visscher, P. K., Finley, J. \& Vetter, R. S. 1999. House-hunting by honey bee swarms: collective decisions and individual behaviors. Insectes Sociaux, 46, 348-360.

Camazine, S., Deneubourg, J., Franks, N. R., Sneyd, J., Theraulaz, G. \& Bonabeau, E. 2001. Self-organization in Biological Systems. Princeton: Princeton University Press.

Conover, W. J. 1999. The Friedman test. In: Practical Nonparametric Statistics (Ed. by W. J. Conover), pp. 369-373. New York: J. Wiley.

Conradt, L. 1998. Could asynchrony in activity between the sexes cause intersexual social segregation in ruminants? Proceedings of the Royal Society of London, Series B, 265, 1359-1363.

Conradt, L. \& Roper, T. J. 2003. Group decision-making in animals. Nature, 421, 155-158.

Conradt, L. \& Roper, T. J. 2005. Consensus decision making in animals. Trends in Ecology and Evolution, 20, 449-456.

Couzin, I. D. \& Franks, N. R. 2003. Self-organized lane formation and optimized traffic flow in army ants. Proceedings of the Royal Society of London, Series B, 270, 139-146.

Couzin, I. D., Krause, J., Franks, N. R. \& Levin, S. A. 2005. Effective leadership and decision-making in animal groups on the move. Nature, 433, 513-516.

Davis, J. H. 1992a. Introduction to the special issue on group decisionmaking organizational behavior and human decision processes. Organizational Behavior and Human Decision Processes, 52, 1-2.

Davis, J. H. 1992b. Some compelling intuitions about group consensus decisions, theoretical and empirical research, and interpersonal aggregation phenomena: selected examples, 19501990. Organizational Behavior and Human Decision Processes, 52, 3-38.

Dornhaus, A., Franks, N. R., Hawkins, R. M. \& Shere, H. N. S. 2004. Ants move to improve: colonies of Leptothorax albipennis emigrate whenever they find a superior nest site. Animal Behaviour, 67, 959-963.

Eliaza, K., Rayb, D. \& Razinc, R. 2007. Group decision-making in the shadow of disagreement. Journal of Economic Theory, 132, 236-273.

Franks, N. R., Pratt, S. C., Mallon, E. B., Britton, N. F. \& Sumpter, D. J. T. 2002. Information flow, opinion polling and collective intelligence in house-hunting social insects. Philosophical Transactions of the Royal Society of London, Series B, 357, 1567-1583.

Franks, N. R., Dornhaus, A., Fitzsimmons, J. P. \& Stevens, M. 2003. Speed versus accuracy in collective decision making. Proceedings of the Royal Society of London, Series B, 270, 24572463.

Helbing, D., Farkas, I. \& Vicsek, T. 2000. Simulating dynamical features of escape panic. Nature, 407, 487-490.

Kils, U. 1986. Verhaltensphysiologische Untersuchungen an pelagischen Schwärmen, Schwarmbildung als Strategie zur Orientierung in Umweltgradienten, Bedeutung der Schwarmbildung in der Aquakultur. Berlin Institute Meereskunde, Kiel, 163, 1-168. 
Kocher, M. G. \& Sutter, M. 2005. The decision maker matters: individual versus group behaviour in experimental beauty-contest games. Economic Journal, 115, 200-223.

Köhler, D. 1976. The interaction between conditioned fish and naive schools of juvenile carp (Cyprinus carpio, Pisces). Behavioural Processes, 1, 267-275.

Krause, J. \& Ruxton, G. D. 2002. Living in Groups. Oxford: Oxford University Press.

Latané, B. \& Wolf, S. 1981. The social impact of majorities and minorities. Psychological Review, 88, 438-453.

Leca, J. B., Gunst, N., Thierry, B. \& Petit, O. 2003. Distributed leadership in semifree-ranging capuchin monkeys. Animal Behaviour, 66, 1045-1052.

Levine, J. M. \& Moreland, R. L. 1990. Progress in small group research. Annual Review of Psychology, 41, 585-634.

Lovvorn, J. R. \& Kirkpatrick, C. M. 1982. Field use by staging Eastern Greater Sandhill Cranes. Journal of Wildlife Management, 46 99-108.

McGrath, J. E. 1978. Small group research. American Behavioral Scientist, 21, 651-674.

Mallon, E. B., Pratt, S. C. \& Franks, N. R. 2001. Individual and aggregated decision making during nest site selection by the ant Leptothorax albipennis. Behavioral Ecology and Sociobiology, 50, 352-359.

Maransky, B. P. \& Bildstein, K. L. 2001. Follow your elders: agerelated differences in the migration behavior of broad-winged hawks at Hawk Mountain Sanctuary, Pennsylvania. Wilson Bulletin, 113, 350-353.

Pratt, S. C. 2005. Quorum sensing by encounter rates in the ant Temnothorax albipennis. Behavioral Ecology, 16, 488-496.

Pratt, S. C., Mallon, E. B., Sumpter, D. J. T. \& Franks, N. R. 2002. Quorum sensing, recruitment aggregated decision making during colony emigration by the ant Leptothorax albipennis. Behavioral Ecology and Sociobiology, 52, 117-127.

Prins, H. H. T. 1996. Ecology and Behaviour of the African Buffalo. London: Chapman \& Hall.

Radakov, D. V. 1973. Schooling in the Ecology of Fish. Translated from the Russian by H. Mills. A Halsted Press Book. New York: J. Wiley.
Reader, S. M. \& Laland, K. N. 2000. Diffusion of foraging innovations in the guppy. Animal Behaviour, 60, 175-180.

Reader, S. M., Kendal, J. R. \& Laland, K. N. 2003. Social learning of foraging sites and escape routes in wild Trinidadian guppies. Animal Behaviour, 66, 729-739.

Reebs, S. G. 2000. Can a minority of informed leaders determine the foraging movements of a fish shoal? Animal Behaviour, 59, 403-409.

Ruckstuhl, K. E. 1998. Foraging behaviour and sexual segregation in bighorn sheep. Animal Behaviour, 56, 99-106.

Ruckstuhl, K. E. \& Neuhaus, P. 2000. Sexual segregation in ungulates: a new approach. Behaviour, 137, 361-377.

Ruckstuhl, K. E. \& Neuhaus, P. 2002. Sexual segregation in ungulates: a comparative test of three hypotheses. Biological Reviews, 77, 77-96.

Seeley, T. D. 2003. Consensus building during nest-site selection in honey bee swarms: the expiration of dissent. Behavioral Ecology and Sociobiology, 53, 417-424.

Seeley, T. D. \& Buhrman, S. C. 1999. Group decision making in swarms of honey bees. Behavioral Ecology and Sociobiology, 45, 19-31.

Seeley, T. D. \& Visscher, P. K. 2004. Group decision making in nestsite selection by honey bees. Apidologie, 35, 101-116.

Stewart, K. J. \& Harcourt, A. H. 1994. Gorillas vocalizations during rest periods - signals of impending departure. Behaviour, 130, 29-40.

Swaney, W., Kendal, J., Capon, H., Brown, C. \& Laland, K. N. 2001. Familiarity facilitates social learning of foraging behaviour in the guppy. Animal Behaviour, 62, 591-598.

Ten Velden, F. S., Beersma, B. \& De Dreu, C. K. W. 2007. Majority and minority influence in group negotiation: the moderating effects of social motivation and decision rules. Journal of Applied Psychology, 92, 259-268.

Van Vugt, M. 2006. Evolutionary origins of leadership and followership. Personality and Social Psychology Review, 10, 354-371.

Winquist, J. R. \& Larson, J. R., Jr. 1998. Information pooling: when it impacts group decision making. Journal of Personality and Social Psychology, 74, 371-377. 